\title{
The power of mass spectrometry in biological discovery
}

\author{
BioTechniques 40:779 (June 2006)
}

doi 10.2144/000112199

Mass spectrometry has had an enormous effect on the capability for structural analysis of molecules for decades. A key step is the ability to create gas phase ions of the molecules to be analyzed. For most organic molecules, it is straightforward to volatize molecules into the high vacuum ion source of mass spectrometers. Thirty to thirty-five years ago, efforts were made to use mass spectrometers to characterize biomolecules. This class of molecule is often charged and polar, making volatilization into an ion source difficult. Solutions were found through chemical derivatization of molecules to eliminate charge and polarity. Of course, chemical derivatization usually decreases sensitivity, requiring adequate starting material and often multiple steps. New ionization techniques that could create ions of biomolecules improved analysis. Plasma desorption was the first of the soft ionization techniques that created ions of biomolecules without fragmentation. Plasma desorption used Californium-252 to create a source of high energy particles to affect desorption, which was not particularly practical. Fast atom bombardment (FAB) brought biomolecular analysis into the mainstream, as the technique could be adapted to a wide variety of mass spectrometers and was effective at creating ions of small biomolecules $(<10,000 \mathrm{Da})$. A disadvantage to $\mathrm{FAB}$, the bombardment of a liquid matrix (such as glycerol)-embedded sample with high energy ions ( $\left.\mathrm{Cs}^{+}\right)$or atoms (Xenon), is the inability to readily couple FAB with liquid chromatography. The revolution in biomolecular analysis arrived about 7 years after the introduction of FAB, with the development of electrospray ionization (ESI) and matrix-assisted laser desorption/ionization (MALDI). The impact of these two new ionization techniques could hardly have been anticipated, as their availability exponentially increased the capability for biomolecule analysis. As is typically the case, new developments in mass spectrometry technology were driven to take advantage of this new capability. Significantly impacted by the creation of a robust interface for liquid chromatography was the use of tandem mass spectrometry for peptide sequence analysis.

Two additional improvements in mass spectrometry was the introduction of instrument control language (ICL) and large-scale data analysis strategies. ICL was an important contribution, as it allowed the creation of automated and intelligent data acquisition strategies to collect tandem mass spectra in large-scale. The collective genome sequencing projects created sequence resources that allowed the development of computer algorithms for the large-scale interpretation of tandem mass spectra. These developments allowed the creation of bottom-up strategies for the analysis of digested protein mixtures commonly termed shotgun proteomics. Shotgun proteomics has been widely applied to a variety of biological discovery problems from the analysis of protein complexes to the search for biomarkers in human serum. In this special section of BioTechniques, three papers describe current developments in proteomics, and Professor John Fenn provides a personal perspective.

Jensen and colleagues provide a review on the identification of posttranslational modifications. Posttranslational modifications are critical components of the cell regulatory machinery. Given the smaller than anticipated number of human genes, functional diversity must be created through other means. One such strategy is controlling activity and localization through alternate splicing and covalent modifications. Deciphering the roles of modifications on proteins requires that we have efficient and sensitive methods to identify modifications of proteins to place them in their biological context. Both the development of methods to discover modifications and the application of these methods to discovery are important ventures.

To improve the discovery of modifications, the development of new mass spectrometry approaches that assist in this activity can have a significant impact. Coon and colleagues describe a new approach in ion trap mass spectrometers to dissociate (fragment) ions with enormous potential to assist in the assignment of modification sites and alternate splicing. Electron transfer dissociation (ETD) and ion/ion reactions allow the dissociation and study of larger ions in mass spectrometers, because one can fragment multiply charged ions of large polypeptides and manipulate the charge states of the resulting fragment ions. Such techniques allow the study of larger polypeptides and even proteins that make it easier to put modifications and alternative splice forms into biological context. The development of new dissociation methods coupled with ion/ion reactions represent an exciting new area in mass spectrometry that will have benefits to proteomics.

The last area discussed is the development of the clinical side of proteomics. There is a tremendous need for diagnostic tests for almost all diseases. Proteomics is viewed as a promising new approach to speed the discovery of proteins that correlate with disease. Of course, most clinical tests are performed on blood, and thus the search for biomarkers has focused on the use of serum or plasma as the source for biomarkers. Serum and plasma represent the most challenging of the body fluids, with a very large range of protein abundances. This situation has challenged the field of proteomics to develop new strategies and techniques to deal with this enormous hurdle. In the third review, Conrads et al. discuss the different strategies in use to discover biomarkers and detail some of the pre-analytic issues that must be considered in the search for biomarkers.

The reviews published in this special section represent a snapshot of ongoing research in the proteomics field. Proteomics is a dynamic and technologically driven field that has accelerated the pace of discovery in biology. These early successes in the field of biology have led to an expansion of applications in clinical medicine. The ability to associate protein expression, modifications, or isoforms to disease will assist in the discovery of disease markers that will be useful for diagnosis and drug development. 